\title{
3-28-2018
}

\section{Blood rheological abnormalities in sickle cell anemia.}

\author{
Philippe Connes \\ University of Lyon; PRES Sorbonne Paris Cité
}

Céline Renoux

University of Lyon; PRES Sorbonne Paris Cité; Hospices Civils de Lyon

Marc Romana

PRES Sorbonne Paris Cité; Hôpital Ricou

Manouk Abkarian

Université de Montpellier

Philippe Joly

University of Lyon; PRES Sorbonne Paris Cité; Hospices Civils de Lyon

Follow this and additional works at: https://jdc.jefferson.edu/medfp

See next page for additional authors

Part of the Hematology Commons

Let us know how access to this document benefits you

\section{Recommended Citation}

Connes, Philippe; Renoux, Céline; Romana, Marc; Abkarian, Manouk; Joly, Philippe; Martin, Cyril; Hardy-Dessources, Marie-Dominique; and Ballas, Samir K., "Blood rheological abnormalities in sickle cell anemia." (2018). Department of Medicine Faculty Papers. Paper 240.

https://jdc.jefferson.edu/medfp/240

This Article is brought to you for free and open access by the Jefferson Digital Commons. The Jefferson Digital Commons is a service of Thomas Jefferson University's Center for Teaching and Learning (CTL). The Commons is a showcase for Jefferson books and journals, peer-reviewed scholarly publications, unique historical collections from the University archives, and teaching tools. The Jefferson Digital Commons allows researchers and interested readers anywhere in the world to learn about and keep up to date with Jefferson scholarship. This article has been accepted for inclusion in Department of Medicine Faculty Papers by an authorized administrator of the Jefferson Digital Commons. For more information, please contact: JeffersonDigitalCommons@jefferson.edu. 


\section{Authors}

Philippe Connes, Céline Renoux, Marc Romana, Manouk Abkarian, Philippe Joly, Cyril Martin, MarieDominique Hardy-Dessources, and Samir K. Ballas 


\section{Blood rheological abnormalities in sickle cell anemia}

Philippe Connes ${ }^{1,2,3}$, Céline Renoux ${ }^{1,2,4}$, Marc Romana ${ }^{2,5}$, Manouk Abkarian ${ }^{6}$, Philippe Joly $^{1,2,4}$, Marie-Dominique Hardy-Dessources ${ }^{2,5}$, Samir K Ballas ${ }^{7}$.

${ }^{1}$ Laboratoire LIBM EA7424, Team"Vascular Biology and Red Blood Cell", Université Claude Bernard Lyon 1, 69100 Villeurbanne, France; ${ }^{2}$ Laboratory of Excellence GR-Ex «The red cell: from genesis to death», PRES Sorbonne Paris Cité, 75015, Paris, France; ${ }^{3}$ Institut Universitaire de France, Paris, France; ${ }^{4}$ Laboratoire de biochimie des pathologies érythrocytaires, Centre de Biologie Est, Hospices Civils de Lyon, France; ${ }^{5}$ Inserm UMR 1134, Hôpital Ricou, CHU de Pointe-à-Pitre, 97157 Pointe-à-Pitre, Guadeloupe; ${ }^{6}$ CNRS UMR 5048, Université de Montpellier, Centre de Biochimie Structurale, 34090 Montpellier, France; ${ }^{7}$ Department of Medicine, Cardeza Foundation for Hematologic Research, Jefferson Medical College, Thomas Jefferson University, Philadelphia, Pennsylvania, USA

\section{Corresponding author}

Philippe Connes, $\mathrm{PhD}$

Laboratoire LIBM EA7424, Team"Vascular Biology and Red Blood Cell”, Université Claude Bernard Lyon 1, 69100 Villeurbanne, France

Email: pconnes@yahoo.fr / philippe.connes@univ-lyon1.fr 


\begin{abstract}
This review focuses on the contribution of abnormal blood rheology in the pathophysiology of sickle cell anemia (SCA). SCA is characterized by a reduction of red blood cell (RBC) deformability but this reduction is very heterogeneous from one patient to another. Recent works have shown that patients with the lowest RBC deformability (measured by ektacytometry) have enhanced hemolysis and would be more prone to develop several complications such as priapism, leg ulcers and glomerulopathy. In contrast, patients with the highest deformability, and not under hydroxyurea therapy, seem to develop more frequently vaso-occlusive like events. Although less studied, RBC aggregation properties are very different between SCA and healthy individuals and it was demonstrated that increased RBC aggregates strength could be involved in some complications. Finally, several works demonstrated that the vascular system of SCA patients could not fully compensate any increase in blood viscosity because of the loss of vascular reactivity, which may result in vaso-occlusive crises.
\end{abstract}

Key words: sickle cell anemia, blood viscosity, red blood cell deformability, red blood cell aggregation 


\section{Introduction}

Sickle cell anemia (SCA) is the most frequent genetic disease in the world. It is characterized by a single nucleotide mutation (adenine $\rightarrow$ thymine) in exon I of the $\beta$-globin gene that leads to the presence of sickle hemoglobin $(\mathrm{HbS})$ resulting from the substitution of valine for glutamic acid at the sixth position of the $\beta$-globin chain. When deoxygenated, $\mathrm{HbS}$ is able to polymerize, hence causing a mechanical distortion of red blood cells (RBCs): i.e., sickling. Sickle RBCs are rigid and do not easily flow through the microcirculation, causing frequent vaso-occlusive episodes in affected patients. Recurrent $\mathrm{HbS}$ polymerization leads to numerous $\mathrm{RBC}$ and systemic physiological abnormalities with variable phenotypic severity.\{Embury, $1986 \# 2\}$ In addition, sickle RBCs are very fragile, which may explain why patients are chronically anemic.

\section{RBC deformability in SCA}

Mean RBC deformability (measured by ektacytometry) is decreased in SCA patients at steady state compared to healthy individuals (Ballas et al, 1988; Messmann et al, 1990; Tripette et al, 2009). The proportion of dehydrated irreversible sickle RBCs (ISCs) in the blood of the patients impact directly on the mean deformability of the whole RBC suspension (Ballas et al, 1992). Both RBC membrane stiffening and increased internal viscosity are responsible for the lowering of RBC deformability (Chien et al, 1970; Li et al, 2017). Deoxygenation of $\mathrm{HbS}$, as it may occur in post-capillary venules, further causes a reduction in RBC deformability because the formation of polymerized $\mathrm{HbS}$ fibers disrupts cell morphology (Chien et al, 1970). When re-oxygenated, most of the sickled RBCs unsickle and recover their deformability, although still reduced compared to healthy RBCs.

2-1. Reduced RBC deformability, increased hemolysis and endothelial dysfunction: 
RBC deformability has been extensively studied in the context of SCA. It appears that patients with leg ulcers (Connes et al, 2013; Bowers et al, 2016), frequent priapism (Cita et al, 2016) or glomerulopathy (Lamarre et al, 2014) have lower RBC deformability than patients without these complications. These findings are in agreement with those of Bartollucci et al (2012) who reported higher number of dense RBCs in patients with leg ulcers, renal dysfunction and priapism. Interestingly, these complications belong to the so-called hemolytic-endothelial dysfunction phenotype (Kato et al, 2007) and we recently reported a negative association between RBC deformability and several markers of hemolysis (Connes et al, 2014). Indeed, patients with a severe reduction in RBC deformability would be characterized by increased hemolytic rate and marked anemia (Connes et al, 2014). Nevertheless, further works are needed to investigate the mechanisms that link reduced RBC deformability and increased RBC fragility in SCA. It is now admitted that hemolysis plays a key role in the pathophysiology of SCA (Kato et al, 2007). Part of the free hemoglobin released into plasma quickly reacts with haptoglobin and the complex is cleared from plasma. However, this system is insufficient to eliminate the excess of free circulating hemoglobin in SCA and a large amount reacts with nitric oxide (NO) to produce methemoglobin and nitrate ultimately leading to reduced NO bioavailability.\{Kato, 2007 \#35\} The release of RBC arginase into the plasma catabolizes plasma arginine reducing its bioavailability. In addition, heme and heme-iron dissociate from $\mathrm{Hb}$ catalyzing the production of reactive oxygen species (ROS) that serve as potent NO scavengers.\{Kato, 2007 \#35\} The decline in blood NO content leads to endothelial dysfunction, over-expression of vascular adhesion molecules and impaired vasomotor tone (Kato et al, 2005; Lin et al, 2005; Moeckesch et al, 2017). In support of these mechanisms, plasma from patients with SCA contains cell-free ferrous oxyhemoglobin, which stoichiometrically consumes micromolar quantities of NO and abrogates forearm blood flow response to NO donor infusion.\{Reiter, $2002 \# 41\}$ In 
summary, it seems that a direct link exists between reduced RBC deformability, increased hemolysis and the development of chronic vasculopathy in SCA (Connes et al, 2016).

\section{2-2. RBC deformability and vaso-occlusive events:}

In contrast, it has been demonstrated that although an increase of RBC deformability is beneficial for blood flow (because of the reduction of blood viscosity for instance) and tissue perfusion (because deformable RBC can easily negotiate small capillaries) in the healthy population, SCA patients with the highest RBC deformability would be prone to osteonecrosis \{Lemonne, 2013 \#60\} and frequent acute painful vaso-occlusive crises (VOC).\{Lamarre, 2012 \#53;Lande, 1988 \#51\} The increased risk of SCA patients with alpha-thalassemia to develop frequent VOC compared to those without has been attributed to the effects of alpha-thalassemia on RBC deformability (Renoux et al, 2017). While RBC deformability is decreased during vaso-occlusive events, Ballas et al.\{Ballas, 1992 \#22\} demonstrated that high level of RBC deformability during the recovery phase of a painful vaso-occlusive event was a predictor of a new painful crisis. Lande et al.\{Lande, 1988 \#51\} also reported a significant and positive correlation between the incidence of painful crisis in SCA and RBC deformability, a result initially reported by Ballas et al.\{Ballas, 1991 \#62;Ballas, 1988 \#61\} This surprising finding may be explained by the fact that sickle RBCs with the highest deformability are also the most adherent RBCs to the vascular wall, thus decreasing the lumen of microvessels, slowing blood flow and initiating vascular occlusion.\{Ballas, 1988 \#61;Kaul, 2004 \#64;Mohandas, 1989 \#63\} The increased adhesiveness of sickle RBCs with the highest deformability would be explained by the fact that they could spread their adhesive patch to a greater extent than the more rigid sickle RBCs (Mohandas et al, 1989). In contrast, although hydroxyurea therapy improves RBC deformability in SCA patients, it also decreases the frequency of VOC-like events (Lemonne 
et al, 2015; In press) because it also decreases the ability of sickle RBCs to adhere to the endothelium (Ware et al, 2010). The deformability of RBCs in SCA is influenced by several factors such as the amount of fetal hemoglobin ( $\mathrm{HbF}$, Lemonne et al, 2015; Renoux et al, 2016), the magnitude of oxidative stress (Hierso et al, 2014) and the level of nitric oxide (Mozar et al, 2016; Wajih et al, 2017).

\section{RBC aggregation in SCA}

RBC aggregation properties have been poorly studied in SCA but several studies reported that $\mathrm{RBC}$ aggregation (which depends on the number of $\mathrm{RBC}$ aggregates and time for $\mathrm{RBC}$ aggregates formation) is rather decreased in SCA patients compared to healthy individuals. The lower RBC aggregation seems to be due to the low ability of the rigid ISCs to form aggregates.\{Chien, 1970 \#8;Connes, 2014 \#21\} Deoxygenating sickle cell blood in vitro results in a further decrease of RBC deformability and RBC aggregation. \{Bucherer, 1992 \#110\} However, patients with increased RBC aggregation seems to be prone to frequent VOC-like events (Lemonne et al, 2012; Renoux et al, 2017). While RBC aggregation is rather decreased in SCA, once formed, sickle RBC aggregates are 2 to 3 fold more robust than healthy RBC aggregates.\{Tripette, 2009 \#15;Waltz, 2012 \#20\} Chronic enhanced oxidative stress has been reported to strongly modulate the strength of RBC aggregates (Hierso et al, 2014). The findings of Loiseau et al (2015) suggest that these sticky RBC aggregates would preferentially deposit at bifurcation level in vascular network, which could increase the risks for vaso-occlusive like events. Indeed, Lamarre et al (2012) found an association between RBC aggregates strength and acute chest syndrome in SCA. No study to date measured the changes in RBC aggregation during a vaso-occlusive event in SCA and only limited data is available on erythrocyte sedimentation rate, a parameter that is affected by both hematocrit and RBC aggregation.\{Lawrence, 1986 \#30\}' \{Awodu, 2009 \#31\} The increase of 
fibrinogen during VOC could promote RBC aggregation\{Awodu, 2009 \#31\} but further studies are needed to directly investigate this parameter during crisis.

\section{Blood viscosity in SCA}

Owing to the chronic severe anemia, patients with SCA have lower blood viscosity at native hematocrit under oxygenated conditions than individuals with normal hemoglobin.\{Tripette, $2009 \# 15\}$ However, blood viscosity increases with ageing in SCA patients (Renoux et al, 2015), which could explain why osteonecrosis and retinopathy prevalence increase with age in this population $\{$ Lemonne, 2014 \#9;Serjeant, $1995 \# 26$ \}. When adjusted to a normal hematocrit levels (40-45\%), SCA patients have blood viscosity that is above the controls under oxygenated conditions (partly due to the presence of ISCs). When deoxygenated, the sickling of RBCs causes a further rise in blood viscosity at all shear rates. $\{$ Chien, $1970 \# 8\}$ Classically, it is suspected that any increase in blood viscosity could be deleterious for the cardiovascular system. However, a rise in blood viscosity is pretty well tolerated in the general population (Salazar-Vazquez et al, 2008). The production of NO by the endothelial NO-synthase is strongly dependent on the shear stress applied on the vascular wall. Shear stress is the product of shear rate by blood viscosity. Indeed, when blood viscosity increases, endothelial cells produce more $\mathrm{NO}$, which results in compensatory vasodilation that normalizes vascular resistance and blood flow (Tsai et al, 2005; Connes et al, 2013). However, in case of endothelial/vascular dysfunction, like in SCA (Kato et al, 2007; Charlot et al, 2016; Ranque et al, 2016; Ataga et al, 2016; Moeckesh et al, 2017), a rise in blood viscosity could not be fully compensated and is suspected to impair blood flow (Kato et al, 2007).

A large epidemiological study in the United States (the Cooperative Study of Sickle Cell Disease) demonstrated that high hematocrit and $\mathrm{Hb}$ increased the risks for VOC \{Platt, 1991 
\#49\} and acute chest syndrome, $\{$ Castro, $1994 \# 50\}$ respectively, in SCA. This is in agreement with previous findings showing that $\mathrm{Hb}$ value is an independent predictor for the development of acute pain crisis in SCA.\{Lande, 1988 \#51\} Hematocrit is a key determinant of blood viscosity, particularly at low shear rate where the rise in viscosity is exponential. Indeed, it is not surprising to observe that SCA patients with more frequent hospital admissions for VOC have higher blood viscosity at steady state than patients with less frequent hospitalizations (Nebor et al, 2011; Lamarre et al, 2012; Renoux et al, 2017). Moreover, blood viscosity was shown to rise further during a vaso-occlusive episode, which may worsen tissue perfusion and oxygenation.\{Awodu, $2009 \# 31$ \}

\section{Conclusion}

Progress has been made in the last decades in the understanding of the pathophysiological mechanisms involved in SCA. Previous and recent studies identified several potential biomarkers and hemorheological predictors of various acute and chronic complications that paved the way for stimulating novel approaches to develop appropriate therapies. In addition, further studies are needed to better understand the relationships between RBC rheological properties, RBC fragility (i.e., hemolysis) and RBC adhesiveness in the context of SCA.

\section{References}


